\title{
An Adaptive Algorithm for Differentially Coherent Detection in the Presence of Intersymbol Interference
}

\author{
Milica Stojanovic, Member, IEEE
}

\begin{abstract}
An adaptive equalization method is proposed for use with differentially coherent detection of M-ary differential phase-shift keying (DPSK) signals in the presence of unknown carrier frequency offset. A decision-feedback or a linear equalizer is employed, followed by the differentially coherent detector. The equalizer coefficients are adjusted to minimize the post-detection mean squared error. The error, which is a quadratic function of the equalizer vector, is used to design an adaptive algorithm of stochastic gradient type. The approach differs from those proposed previously, which linearize the post-detection error to enable the use of least mean squares (LMS) or recursive least squares (RLS) adaptive equalizers. The proposed quadratic-error $(Q)$ algorithm has complexity comparable to that of LMS, and equal convergence speed. Simulation results demonstrate performance improvement over methods based on linearized-error $(L)$ algorithm. The main advantages of the technique proposed are its simplicity of implementation and robustness to carrier frequency offset, which is maintained for varying modulation level.
\end{abstract}

Index Terms-Adaptive equalization, decision-feedback equalization, differentially coherent detection, noncoherent detection, phase tracking, stochastic gradient.

\section{INTRODUCTION}

D IFFERENTIALLY coherent detection offers the simplest way of achieving carrier synchronization with M-ary phase-shift keying (PSK), and, thus, represents an attractive solution for systems in which explicit phase tracking is not an option. However, differentially coherent detection is based on the premise that there is no intersymbol interference (ISI) in the received signal. When a frequency selective multipath channel introduces ISI, differentially coherent detection must be combined with equalization. The question then arises as to how the two techniques should be combined, i.e., whether to equalize first and then perform differentially coherent detection, or to compensate for the unknown, time-varying phase first, and then equalize. Various solutions to this problem have been proposed in the literature, which includes early work by Sehier and Kaleh [1], more recent results by Masoomzadeh-Fard and Pasupathy [2], Colavolpe and Raheli [3], and extensive work by Schober et al. [4]-[6]. Below, we briefly summarize some of the existing solutions.

The method proposed in [1] uses linear equalization (LE) followed by differentially coherent detection. The equalizer optimization criterion is minimization of the mean squared error

Manuscript received March 31, 2004; revised January 27, 2005 and March 30,2005 .

The author is with the Massachusetts Institute of Technology, Cambridge, MA 02139 USA (e-mail: millitsa@mit.edu).

Digital Object Identifier 10.1109/JSAC.2005.853808
(MMSE) obtained after differential detection. However, the post-detection error is a quadratic, rather than a linear function of the equalizer vector, and this fact prevents application of the least mean squares (LMSs) or recursive least squares (RLS) algorithm in the usual manner. To overcome this difficulty, a simplifying assumption is made: equalizer coefficients are taken to be independent from one symbol interval (iteration) to another. Under this assumption, the instantaneous error is linearized, allowing for the application of standard LMS. Effectively, the equalizer is applied to the input signal premultiplied by the conjugate of a previously estimated data symbol, and then adjusted ignoring the fact that there is dependence between its input and output signals. Nonetheless, this algorithm, called the modified LMS, offered acceptable performance in a variety of conditions, and has consequently been used in many later developments, e.g., [2] and [4].

To accommodate severe ISI, in [2], the authors proposed to perform differentially coherent demodulation first, followed by decision-feedback equalization (DFE). However, because the input signal contains ISI, differentially coherent demodulation introduces nonlinear distortion, thus making this approach suboptimal, as demonstrated by the fact that it offered improvement over LE only on severely distorted channels. In addition, the nonlinear equalizer must operate at twice the sampling rate of its linear counterpart (fractional spacing $T / 4$ instead of $T / 2$ ).

Optimal noncoherent detection for channels with ISI is based on maximum-likelihood sequence estimation (MLSE) in the presence of unknown phase. To reduce the inherent complexity of MLSE, techniques based on reduced-state Viterbi algorithm were proposed in [3] for time-invariant ISI channels. Adaptive schemes were addressed in [4] for linear, and in [5] for nonlinear equalization. These nonlinear schemes are optimal in the sense that they are not applied to a nonlinearly predistorted signal as in [2]; instead, MLSE or DFE are applied to the input signal containing undistorted (linear) ISI as in [3]. To do so, a reference symbol is generated from multiple past observations (recursively or nonrecursively) and used to correct the phase of the incoming signal prior to adaptive equalization. Because these methods essentially generate a carrier phase reference, they can approach the performance of coherent detection, which occurs in the limit as the number of previous symbol estimates used to generate the reference tends to infinity. The linear equalizer of [4] uses a modified LMS/RLS similarly as [1] and [2]. Adaptation of the MLSE/DFE [5] is guided by the error at the equalizer output (predetection error) and is, thus, decoupled from differentially coherent detection that follows. Consequently, there is a tradeoff between tolerable frequency offset and ISI compensation capability. The M-ary differential 
phase-shift keying (DPSK) principles of [4] and [5] were extended to the case of M-ary differential amplitude/phase-shift keying (DAPSK) in [6].

The method proposed in this paper seeks to improve upon the existing methods by: 1) using a simple receiver structure consisting of a DFE (or LE) followed by the differentially coherent detector and 2) by applying an adaptive MMSE algorithm that makes no simplifying assumptions to linearize the post-detection error. Rather than assuming independence between two consecutive values of the equalizer vector, the opposite is assumed: namely, that the equalizer vector does not change much from one symbol interval to another. Because the task of tracking fast changes in the carrier phase is left to the differentially coherent detector, such an assumption seems intuitively more satisfying. Thus, the equalizer is optimized within the framework of differentially coherent detection, i.e., jointly with it. The resulting algorithm is of stochastic gradient type, and has complexity comparable to that of the modified LMS [1]. When used with the DFE, algorithm performance can be enhanced by preventing the feedback coefficients from rotation. This is necessary only in the presence of a large frequency offset. A method for generating the needed phase reference from the past data symbol estimates is proposed for this situation. This method targets directly the residual phase of the past estimates, and in this manner differs from the recursive phase reference method of [5], which is based on planar filtering of a reference phasor. When only a linear equalizer is used, phase reference is not needed in the receiver proposed.

The present work is motivated by the problem of bandwidthefficient modulation/detection in underwater acoustic wireless channels, where extensive ISI accompanies rapid phase variations [7], but the solution is applicable to any ISI channel with unknown carrier phase. The main advantages of the algorithm proposed are its simplicity, robustness to carrier phase offset, and performance improvement of the adaptive algorithm based on quadratic error over its linearized-error counterpart.

The receiver structure and the adaptive algorithm are presented in Section II. In Section III, performance of the proposed method is assessed through simulation. Conclusions are summarized in Section IV.

\section{ReCEIVER STRUCTURE AND THE ALGORITHM}

The baseband-equivalent received signal is given by

$$
v(t)=\sum_{n} d(n) h(t-n T) e^{j \theta(t)}+z(t)
$$

where $d(n)$ are the transmitted M-ary DPSK data symbols, $T$ is the symbol interval, $h(t)=g_{T}(t) * c(t) * g_{R}(t)$ is the overall system response (including transmit filtering, channel, and receive filtering), $\theta(t)$ is the unknown carrier phase, and $z(t)=$ $w(t) * g_{R}(t)$ is the noise that results from filtering the equivalent baseband input additive white Gaussian noise (AWGN) $w(t)$ whose independent in-phase and quadrature components each have power spectral density $N_{0}$. The data symbols $d(n)$ are obtained by differentially encoding the input M-ary PSK symbols $b(n) \in\left\{1, e^{j 2 \pi / M}, \ldots, e^{j 2 \pi(M-1) / M}\right\}$, i.e., $d(n)=$ $b(n) d(n-1)$.
We assume that the channel is not known, and, hence, instead of a complete matched filter, the receiver uses only a lowpass filter. The transmit and receive filters can both be chosen as square-root raised cosine filters. The received signal is sampled at the Nyquist or higher rate, i.e., every $T_{s}=T / 2$, and the samples are fed into a fractionally spaced equalizer. The vector of signal samples, stored at time $n T$ in the feedforward filter with the coefficient vector $\mathbf{a}^{\prime}$ is denoted by $\mathbf{v}(n) .{ }^{1}$ It is assumed that proper time-alignment has been achieved so that this signal contains significant contributions from the data symbol $d(n)$. The vector of previously detected data symbols, stored at time $n T$ in the feedback filter $\mathbf{b}^{\prime}$, is denoted by $\tilde{\mathbf{d}}(n-1)=[\tilde{d}(n-$ 1) $\tilde{d}(n-2) \ldots]^{T}$, where $[\cdot]^{T}$ stands for transpose. These symbols are either the true data symbols known during training, or the decisions generated by differentially re-encoding the output data stream $\tilde{b}(n)$, i.e., $\tilde{d}(n)=\tilde{b}(n) \tilde{d}(n-1)$. The equalizer output at time $n T$ is the estimate

$$
\hat{d}_{\theta}(n)=\mathbf{a}^{\prime} \mathbf{v}(n)-\mathbf{b}^{\prime} \tilde{\mathbf{d}}(n-1) \Theta(n)=\mathbf{c}^{\prime} \mathbf{y}(n)
$$

where $\mathbf{c}^{\prime}=\left[\mathbf{a}^{\prime}-\mathbf{b}^{\prime}\right]$ is the composite equalizer vector, and $\mathbf{y}(n)$ is the accordingly defined composite signal vector. The factor $\Theta(n)=e^{j \hat{\theta}(n)}$ is needed only in the presence of a significant frequency offset $f_{d}$ (roughly speaking when $f_{d} T>10^{-3}$ ). If only a linear equalizer is used $(\mathbf{b}=\mathbf{0})$, there is no need for this factor. The estimate $\hat{d}_{\theta}(n)$ contains residual phase variation in addition to the desired contribution of the data symbol $d(n)$, and the task of phase correction is left to the differentially coherent detector, which yields the estimate of the data symbol $b(n)$

$$
\hat{b}(n)=\hat{d}_{\theta}(n) \hat{d}_{\theta}^{*}(n-1)=\mathbf{c}^{\prime} \mathbf{y}(n) \mathbf{y}^{\prime}(n-1) \mathbf{c} .
$$

This estimate is used to make the symbol decision $\tilde{b}(n)$ from the M-ary PSK alphabet.

In the presence of a frequency offset, the factor $\Theta(n)$ is introduced to aid the feedback filter by preventing its coefficients $\mathbf{b}$ from rotating. Namely, the feedback term $\mathbf{b}^{\prime} \tilde{\mathbf{d}}(n-1) \Theta(n)$ must be aligned in phase with the feedforward term $\mathbf{a}^{\prime} \mathbf{v}(n)$ to achieve effective post-cursor ISI cancellation. An adaptive equalizer is capable of tracking slow phase variations, but not a significant frequency offset (e.g., [7]). When such an offset is present, the term $\mathbf{a}^{\prime} \mathbf{v}(n)$ contains residual phase variation associated with the input signal $\mathbf{v}(n)$, which may be too fast for the feedback filter to track alone. Hence, rather than trying to absorb the residual phase variation into the feedback coefficients (which would cause them to rotate), the correction is included explicitly.

The simplest way to generate $\Theta(n)$ is directly from the residual phase of the estimates $\hat{d}_{\theta}(n)$. Namely, once the decision on the data symbol $\tilde{d}(n)$ becomes available, it can be used to remove the information content of $\hat{d}_{\theta}(n)$, leaving the residual phase offset

$$
\Theta(n+1)=\frac{\hat{d}_{\theta}(n) \tilde{d}^{*}(n)}{\left|\hat{d}_{\theta}(n) \tilde{d}^{*}(n)\right|} \equiv \Phi(n)
$$

${ }^{1} \mathrm{All}$ the vectors are defined as column vectors, and the prime denotes conjugate transpose. 


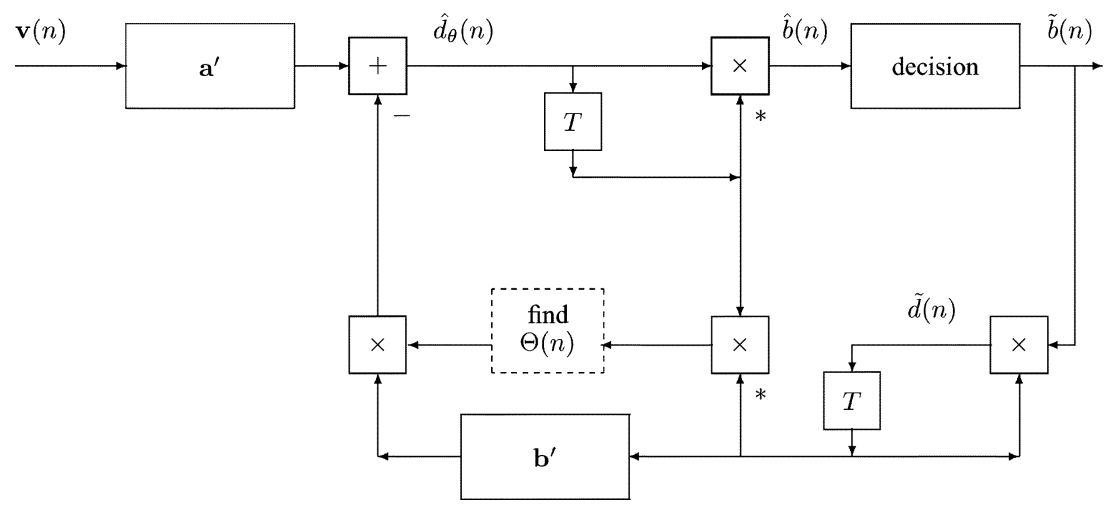

Fig. 1. Block diagram of the receiver. The MMSE equalizer coefficients $\mathbf{a}$ and $\mathbf{b}$ are adjusted adaptively using the post-detection error $e(n)=\tilde{b}(n)-\hat{b}(n)$. Phase correction in the feedback may or may not be necessary; the simplest way to find $\Theta(n)$ is by amplitude limiting (4).

The receiver structure that incorporates these functions is shown in Fig. 1.

\section{A. Improved Feedback Phase Tracking}

An improved estimate of residual phase $\hat{\theta}(n)$ can be obtained by filtering the phase of the term $\Phi(n)$ given in (4). More precisely, since it is only the estimate of frequency offset that is needed, we focus on filtering the phase difference $\Delta \phi(n)=$ $\angle \Phi(n) \Phi^{*}(n-1)$. An exponential weighting filter is given by

$$
\Delta \hat{\theta}(n+1)=\lambda \Delta \hat{\theta}(n)+(1-\lambda) \Delta \phi(n)
$$

where $\lambda \in[0,1)$ and the factor $(1-\lambda)$ ensures an unbiased estimate. The desired phase is

$$
\hat{\theta}(n+1)=\hat{\theta}(n)+\Delta \hat{\theta}(n+1)
$$

with $\hat{\theta}(0)=\Delta \hat{\theta}(0)=0$. Note that for $\lambda=0$, the above expressions reduce to (4).

A computationally simpler alternative to explicit phase extraction is to estimate the instantaneous frequency offset as

$\Delta \hat{\phi}(n)=\operatorname{Im}\left\{\frac{\Phi(n)-\Phi(n-1)}{\Phi(n-1)}\right\}=\operatorname{Im}\left\{\Phi(n) \Phi^{*}(n-1)\right\}$

and use this estimate instead of $\Delta \phi(n)$ in (5). An even simpler frequency estimate results if the amplitude of the data estimate $\hat{d}_{\theta}(n)$ is approximated as being close to 1 . Then, $\Delta \hat{\phi}(n) \approx$ $\operatorname{Im}\left\{\hat{b}(n) \tilde{b}^{*}(n)\right\}$, which may be justified for high signal-to-noise ratio (SNR).

We emphasize that feedback phase tracking is necessary only in the presence of significant frequency offset. The filtering method proposed above enables fast tracking necessary in such a situation, by operating directly on the phase of the signal $\Phi(n)$. Simulation results indicate that additional filtering is particularly beneficial for channels with severe ISI.

\section{B. Equalizer Adaptation}

The error at the output of the differentially coherent detector (post-detection error) can be expressed as

$$
e(n)=b(n)-\hat{b}(n)=b(n)-\mathbf{c}^{\prime} \mathbf{Y}(n) \mathbf{c}
$$

where $\mathbf{Y}(n)=\mathbf{y}(n) \mathbf{y}^{\prime}(n-1)$. This error is to be used to determine the equalizer vector $\mathbf{c}$ according to the MMSE criterion.
When the symbol decisions are correct and additional phase correction is not performed, $\mathbf{Y}(n)$ is independent of $\mathbf{c}$. When the phase correction factor is used, it is treated as independent from equalization. Differentiating the MSE $E=E\left\{\left|e^{2}(n)\right|\right\}$ with respect to the equalizer vector gives the error gradient

$$
\frac{\partial E}{\partial \mathbf{c}^{*}}=-E\left\{\left[e^{*}(n) \mathbf{Y}(n)+e(n) \mathbf{Y}^{\prime}(n)\right] \mathbf{c}\right\} .
$$

Because the error (8) is a quadratic, rather than a linear function of the equalizer vector, there is no apparent closed form solution for the vector $\mathbf{c}$ which sets the gradient to zero. Nonetheless, the solution can be obtained numerically, using the stochastic gradient approach. The resulting algorithm is given by

$$
\begin{aligned}
\hat{b}(n) & =\mathbf{c}^{\prime}(n) \mathbf{Y}(n) \mathbf{c}(n) \\
e(n) & =\tilde{b}(n)-\hat{b}(n) \\
\mathbf{c}(n+1) & =\mathbf{c}(n)+\mu\left[e^{*}(n) \mathbf{Y}(n)+e(n) \mathbf{Y}^{\prime}(n)\right] \mathbf{c}(n)
\end{aligned}
$$

where $\mu$ is the step size, and $\tilde{b}(n)=b(n)$ during training. The algorithm can be initialized by setting the reference feedforward coefficient to 1 , while all the other equalizer coefficients are set to 0 . We call this algorithm the quadratic-error (Q) algorithm to signify the fact that the instantaneous MSE gradient takes into account the quadratic error dependence on the equalizer vector.

There are several ways in which the Q algorithm can be implemented. Expressions (10) define one implementation. In this implementation, computational efficiency can be achieved by exploiting the shifting properties of $\mathbf{y}(n)$ to calculate $\mathbf{Y}(n)$ recursively. Another implementation is the following:

$$
\begin{aligned}
\hat{d}_{\theta}(n) & =\mathbf{c}^{\prime}(n) \mathbf{y}(n) \\
\hat{b}(n) & =\hat{d}_{\theta}(n) \hat{d}_{\theta}^{*}(n-1) \\
e(n) & =\tilde{b}(n)-\hat{b}(n) \\
\mathbf{c}(n+1) & =\mathbf{c}(n)+\mu\left[e^{*}(n) \mathbf{y}(n) \hat{d}_{\theta}^{*}(n-1)\right. \\
& \left.+e(n) \mathbf{y}(n-1) \hat{d}_{\theta}^{*}(n)\right] .
\end{aligned}
$$

The two algorithms are identical when $\mathbf{c}(n)$ reaches steadystate. Both algorithms are based on the assumption that $\mathbf{c}(n)$ does not change much from one iteration to another.

It is interesting at this point to draw a parallel with the modified LMS [1], which linearizes the error by assuming that the equalizer vectors $\mathbf{c}(n)$ and $\mathbf{c}(n-1)$ are independent. Differentiating the MSE with respect to $\mathbf{c}(n)$ only, and using 
the stochastic gradient algorithm, effectively results in applying ordinary LMS to the input signal premultiplied by $\hat{d}_{\theta}^{*}(n-1)$. Although the modified LMS was used for LE only [1]-[4], it can be extended to the DFE. This algorithm, which we call the linearized-error (L) algorithm, is given by the same set of equations (11) from which the second term of the gradient vector, $e(n) \mathbf{y}(n-1) \hat{d}_{\theta}^{*}(n)$, has been removed. Thus, the complexity of the $\mathrm{Q}$ algorithm is only slightly greater than that of the $\mathrm{L}$ algorithm.

The convergence and stability analysis of the Q algorithm remain difficult. These issues were addressed to some extent through simulation, which indicates that the common rules of LMS step size selection apply to the Q algorithm as well.

When the equalizer's task is to compensate for slowly varying ISI, while leaving the tracking of faster phase variation to the differentially coherent detector, the assumption that the equalizer coefficients do not change much from one symbol interval to another is intuitively more satisfying than that of their independence. Hence, one expects better performance from the $\mathrm{Q}$ algorithm than the $\mathrm{L}$ algorithm. Simulation results confirm this expectation.

\section{PERformance Results}

Two multipath channels are considered, both modeled by the impulse response $c(t)=c_{0} \delta(t)+c_{1} \delta(t-T)$. The first channel, called channel $\mathrm{A}$, is defined by $c_{1}=0.387 c_{0}$. The second channel, $\mathrm{B}$, is defined by $c_{1}=c_{0}$. The channel coefficients are normalized such that $\left|c_{0}\right|^{2}+\left|c_{1}\right|^{2}=1$, and the transmit/receive filters $g(t)$ are chosen as square-root raised cosine filters with roll-off factor 1 . The average received energy per bit is $E_{b}=E\left\{|d(n)|^{2}\right\} E_{g} / 2 m=E_{g} / 2 m$, where $m$ is the number of bits per symbol ( $2^{m}$ is the modulation level) and $E_{g}$ is the energy of the pulse $g(t)$. For simulation, the discrete-time filter coefficients $g[l]$ were generated over a span of eight symbol intervals, and normalized such that $\sum_{l}|g[l]|^{2}=1$. The variance of the discrete-time input AWGN is obtained as $\sigma_{w}^{2}=$ $N_{0} / m E_{b}$. A $T / 2$ fractionally spaced equalizer is used with five taps in linear configuration, $\operatorname{LE}(5)$, or three feedforward and one feedback tap in decision-feedback configuration, $\operatorname{DFE}(3,1)$. Extending the number of taps beyond these values results in little improvement. The carrier phase is modeled as $\theta(t)=$ $\theta_{0}+2 \pi f_{d} t$, and several values of the normalized frequency offset $f_{d} T$ were considered in simulation. The values of $f_{d} T$ on the order of $10^{-4}$ and $10^{-3}$ can result from the Doppler shift in land mobile systems. The value $10^{-2}$ is very high, and may be considered as an upper limit on practical $f_{d} T$. Exceptions are found in systems such as low Earth orbiting (LEO) satellite systems, where a much greater $f_{d} T$ results from the high velocity of satellites, making differentially coherent detection infeasible.

The Q algorithm is implemented using version (10). There is little degradation in performance of version (11). The $\mathrm{L}$ algorithm is implemented using (11) without the second gradient term. As a benchmark for performance comparison, a coherent receiver is used which consists of the same-size LMS equalizer, and a jointly optimized phase estimator implemented as a second-order, decision-directed phase-locked loop (PLL)
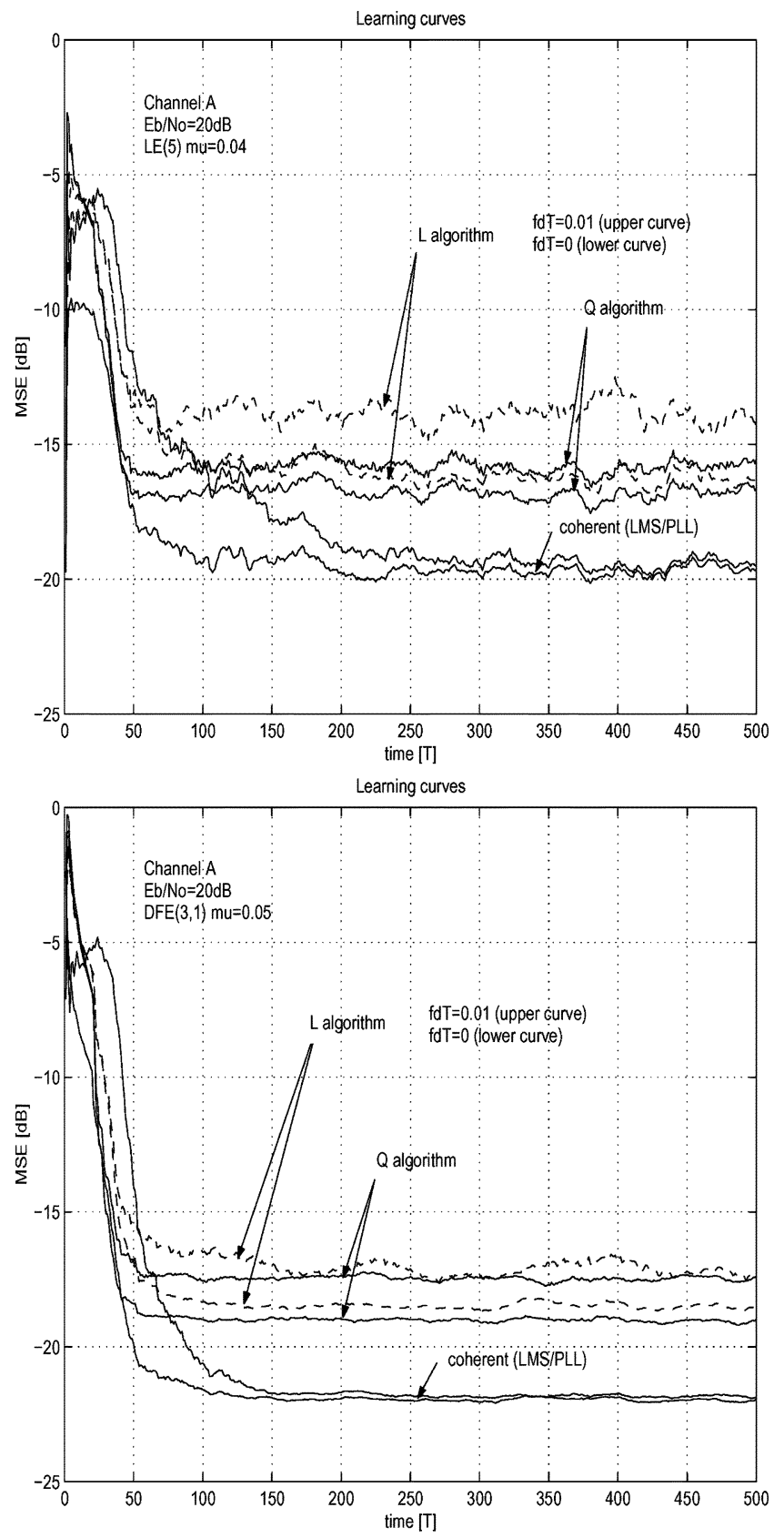

Fig. 2. Learning curves of various algorithms operating on channel A, with 4DPSK, using linear and decision-feedback equalizer.

[7]. The step size $\mu$ is chosen equal for all the receivers as $\mu=0.2 /\left(N\left(1+\sigma_{w}^{2}\right)+M\right)$, where $(N, M)$ is the equalizer size [8]. The initial values of the equalizer coefficients are set to 0 , except with version (10) of the $\mathrm{Q}$ algorithm, when one of the feedforward coefficients is set to a small nonzero value, or 1 , to ensure startup. With these initial conditions, a convergence rate of $100 \%$ was observed in simulation, although there is no analytical proof for the global convergence of either the L algorithm [4] or the Q algorithm.

Fig. 2 illustrates the learning curves of the $\mathrm{Q}$ algorithm. Channel A is used, with 4DPSK, and results are shown for both LE and DFE. Averaging is performed over 1000 simulation runs, with additional smoothing over 20 symbol intervals for easier viewing. Interestingly, we observe that the $\mathrm{Q}$ algorithm 
converges as fast as the ordinary LMS used by the coherent receiver. (No phase tracking is employed with the coherent receiver when $f_{d} T=0$; in all other situations, the PLL proportional and integral tracking constants are set to 0.05 and 0.005 , respectively.) In addition, convergence rate of the $\mathrm{Q}$ algorithm is not affected by the frequency offset (only the steady-state MSE is affected). Results clearly demonstrate that the Q algorithm reaches a lower steady-state MSE than the $\mathrm{L}$ algorithm, and uniformly so across the range of frequency offsets investigated. This is the benefit of optimal design that does not rely on the $\mathbf{c}(n) / \mathbf{c}(n-1)$ independence assumption. The steady-state MSE reached by the Q algorithm at $f_{d} T=0$ is about $3 \mathrm{~dB}$ above that of the coherent receiver. This is the inherent penalty of differentially coherent detection. At $f_{d} T=10^{-2}$, there is an additional loss of about $1 \mathrm{~dB}$, approximately equal for the LE and the DFE configurations. The DFE is implemented here with the simplest form of phase correction (4), i.e., without additional filtering $(\lambda=0)$. The $\mathrm{L}$ algorithm exhibits higher sensitivity to frequency offset in the LE than in the DFE configuration. In the LE configuration, its steady-state MSE at $f_{d} T=0$ is comparable to that of the Q algorithm at $f_{d} T=10^{-2}$.

Probability of error results for channel A and LE are summarized in Fig. 3. Shown are the results for 4DPSK and 8DPSK, each for several values of the normalized frequency offset. Performance is evaluated in the decision-directed mode. The reference curve for coherent detection corresponds to perfectly known frequency offset. At $f_{d} T=0$, there is little difference in performance of the $\mathrm{Q}$ and the $\mathrm{L}$ algorithms. Simulation results for $f_{d} T=10^{-4}$ are practically indistinguishable from those obtained with $f_{d} T=0$. However, as $f_{d} T$ increases, the benefits of the Q algorithm become apparent. With 4DPSK, performance of the $\mathrm{Q}$ algorithm at $f_{d} T=10^{-2}$ differs by less than a decibel from that at $f_{d} T=0$, while performance of the $\mathrm{L}$ algorithm suffers a loss of about $2 \mathrm{~dB}$. Results are also shown for $f_{d} T=0.05$, a case which tests performance limits.

With 8DPSK, there is approximately $4 \mathrm{~dB}$ loss in power efficiency as compared with 4PSK at $f_{d} T=0$, which is on the order of that incurred over an ideal (no ISI) channel. With an increase in the constellation size, it can be expected that an adaptive algorithm will demonstrate increased sensitivity to the frequency offset. Comparing the 8DPSK performance to the 4DPSK performance demonstrates robustness of the $\mathrm{Q}$ algorithm to the increase in modulation level. Namely, as $f_{d} T$ spans the range from 0 to $10^{-2}$, the $\mathrm{Q}$ algorithm suffers about $2 \mathrm{~dB}$ loss within the range of $E_{b} / N_{0}$ shown, while the performance of the $\mathrm{L}$ algorithm saturates.

Fig. 4 shows the performance of DFE on channel A. These results demonstrate the possibility to combine the simple DFE with differentially coherent detection and obtain the expected performance improvement over LE. The DFE improves the performance on this channel by about $2 \mathrm{~dB}$. The DFE, be it with the $\mathrm{Q}$ or the $\mathrm{L}$ algorithm, is aided by the phase tracking method (4). This simplest way of phase tracking is sufficient for the channel at hand. The Q algorithm slightly outperforms the $\mathrm{L}$ algorithm, and both show robustness to frequency offset up to $f_{d} T=10^{-2}$,
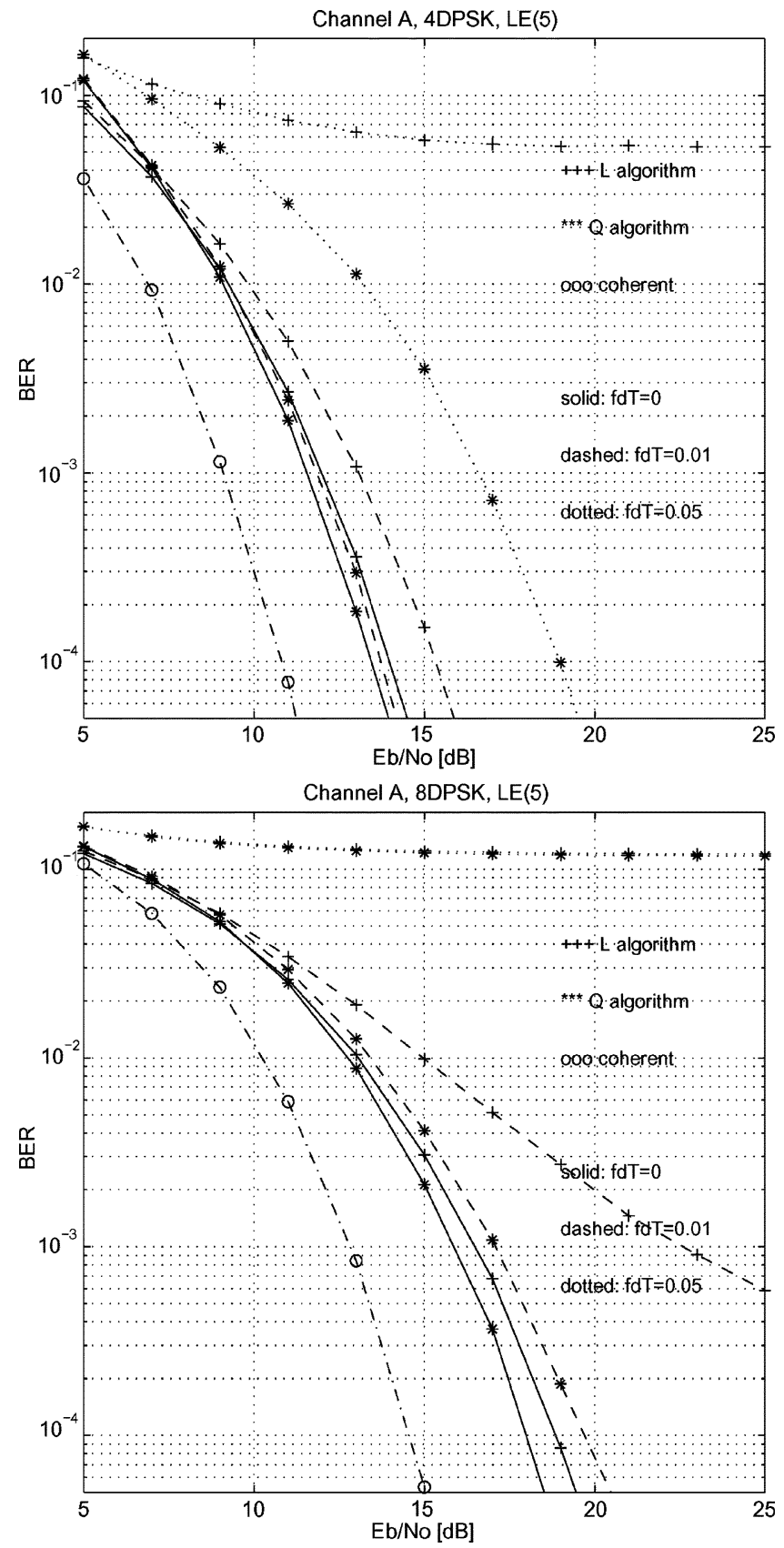

Fig. 3. Probability of bit error as a function of $E_{b} / N_{0}$ for channel A and linear equalization.

as well as to the increase in modulation level. At $f_{d} T=0.05$ (not shown), the $\mathrm{Q}$ algorithm exhibits $5 \mathrm{~dB}$ of additional loss with 4DPSK, while the $\mathrm{L}$ algorithm fails.

Fig. 5 shows the performance results obtained for channel B. Unlike channel A, this channel is not amenable to LE as it has a spectral null. Phase tracking in the feedback section now benefits from additional filtering, which was implemented in the form (5) with $\lambda=0.9$. Explicit phase extraction was employed for the results shown, although the method (7) is equally applicable, as the underlying approximation holds for $f_{d} T \leq 10^{-2}$. It is interesting to note that additional phase filtering is beneficial for channel B, which is explained by the fact that unlike with channel A, performance relies heavily on feedback equalization. 

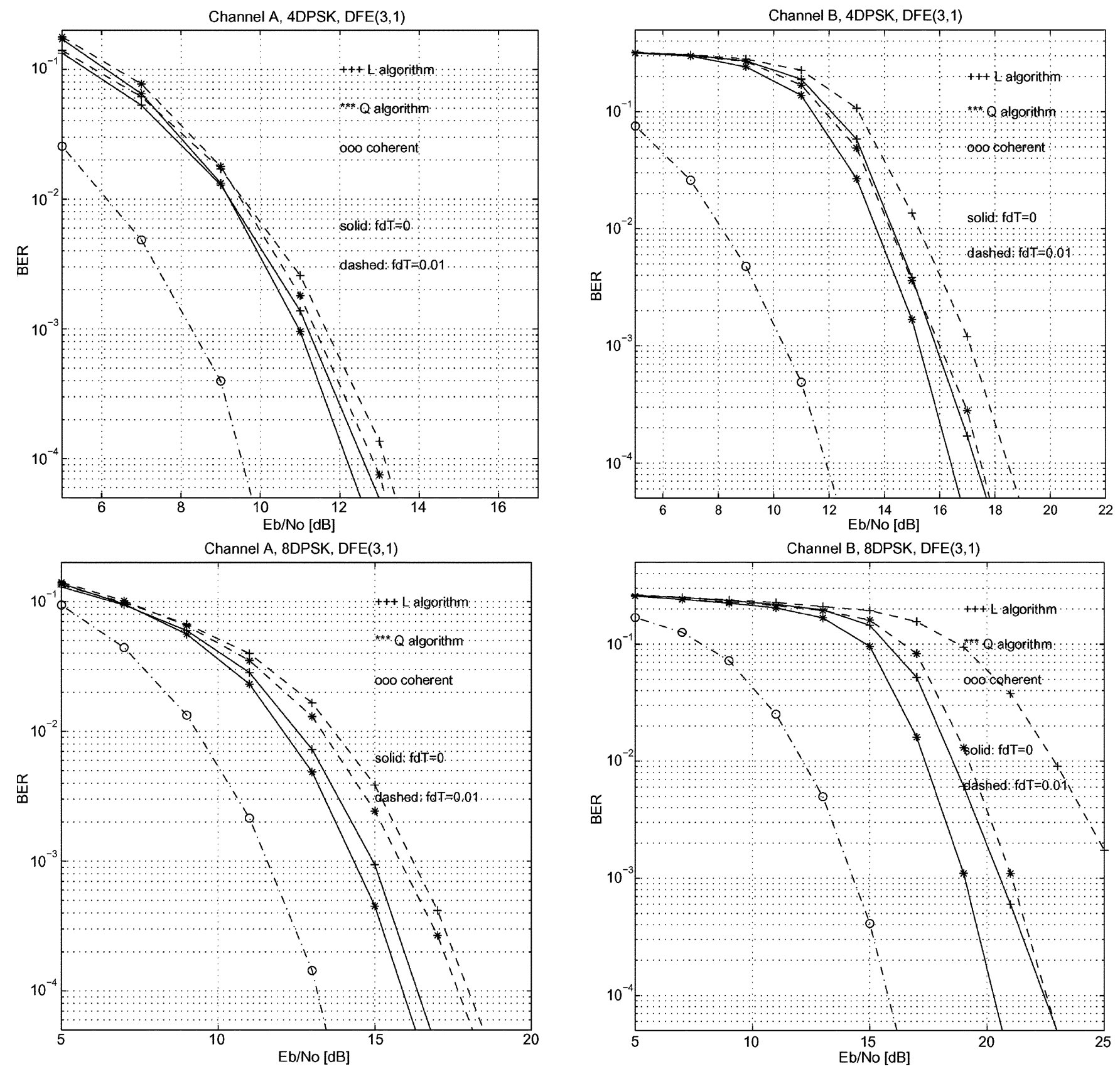

Fig. 4. Probability of bit error as a function of $E_{b} / N_{0}$ for channel A and decision-feedback equalization.

The DFE operating under the Q algorithm provides robust performance for a wide range of frequency offsets on this channel as well. For the SNR range shown, there is less than a decibel degradation between $f_{d} T=0$ and $f_{d} T=10^{-2}$ with 4DPSK (compared with $2 \mathrm{~dB}$ for the $\mathrm{L}$ algorithm) and about $2 \mathrm{~dB}$ degradation with 8DPSK (compared with $6 \mathrm{~dB}$ for the $\mathrm{L}$ algorithm). Thus, the $\mathrm{Q}$ algorithm maintains robustness to frequency offset with varying modulation level in the presence of severe ISI. This fact, together with its implementation simplicity, are the main features of the receiver proposed.

The Q algorithm does not approach performance of coherent detection as do the algorithms of [4] and [5] at small frequency offset. However, the algorithms [4], [5] exhibit a tradeoff be-

Fig. 5. Probability of bit error as a function of $E_{b} / N_{0}$ for channel B and decision-feedback equalization.

tween performance improvement and robustness to frequency offset. In contrast, the $\mathrm{Q}$ algorithm outperforms the $\mathrm{L}$ algorithm consistently over a wide range of frequency offsets. Its practical importance is for applications where fine-tuning of phase tracking parameters, required for coherent detection [7], is not an option.

\section{CONCLUSION}

Differentially coherent detection in the presence of ISI can be accomplished using a DFE (or LE) followed by the differentially coherent detector. To prevent its taps from rotation, the feedback filter of a DFE may be aided by a carrier reference obtained directly from the equalizer output. Filtering the residual phase of 
this signal ensures fast tracking, necessary when significant frequency offset $\left(f_{d} T>10^{-3}\right)$ accompanies severe ISI. In the LE configuration, the carrier reference is not needed. The equalizer coefficients are adjusted to minimize the post-detection MSE. An adaptive algorithm of stochastic gradient type can be designed despite the fact that the error is a quadratic function of the equalizer vector and, hence, there is no apparent closed form MMSE solution. The resulting $\mathrm{Q}$ algorithm outperforms its linearized-error counterpart ( $\mathrm{L}$ algorithm) over a wide range of frequency offsets. This benefit comes from the fact that the equalizer is optimized within the framework of differentially coherent detection, i.e., without the assumption of equalizer vector independence from one symbol interval to another. The complexity of the $\mathrm{Q}$ algorithm is comparable to that of the L algorithm. Simulation results demonstrate convergence speed equal to that of the LMS algorithm, with convergence time not affected by the frequency offset. Probability of error results, obtained for the test channels with and without spectral nulls, demonstrate robust performance, with small penalty for the range of frequency offsets $f_{d} T$ between 0 and $10^{-2}$, as well as low sensitivity to the increase in modulation level from 4 to 8 . While the discussion was presented primarily in terms of the DFE, a practical application may adopt the LE configuration for complexity reasons. Notably, the Q algorithm is suited to this situation, offering performance improvement over the $\mathrm{L}$ algorithm, as well as simplicity of implementation. Future work should capitalize on the benefits offered by the $\mathrm{Q}$ algorithm by extending it to diversity reception as well as to detection of DAPSK signals.

\section{REFERENCES}

[1] P. Sehier and G. K. Kaleh, "Adaptive equalizer for differentially coherent receiver," IEE Proc., pt. 1, vol. 137, no. 1, pp. 9-12, Feb. 1990.
[2] A. Masoomzadeh-Fard and S. Pasupathy, "Nonlinear equalization of multipath fading channels with noncoherent demodulation," IEEE J. Sel. Areas Commun., vol. 14, no. 3, pp. 512-520, Apr. 1996.

[3] G. Colavolpe and R. Raheli, "Noncoherent sequence detection," IEEE Trans. Commun., vol. 47, no. 9, pp. 1376-1385, Sep. 1999.

[4] R. Schober, W. H. Gerstacker, and J. B. Huber, "Adaptive linear equalization combined with noncoherent detection for MDPSK signals," IEEE Trans. Commun., vol. 48, no. 5, pp. 733-738, May 2000.

[5] R. Schober and W. Gerstacker, "Adaptive noncoherent DFE for MDPSK signals transmitted over ISI channels," IEEE Trans. Commun., vol. 48, no. 7, pp. 1128-1140, Jul. 2000.

[6] R. Schober, W. H. Gerstacker, and J. B. Huber, "Adaptive noncoherent linear minimum ISI equalization for MDPSK and MDAPSK signals," IEEE Trans. Signal Process., vol. 49, no. 9, pp. 2018-2030, Sep. 2001.

[7] M. Stojanovic, J. A. Catipovic, and J. G. Proakis, "Phase-coherent digital communications for underwater acoustic channels," IEEE J. Oceanic Eng., vol. 19, no. 1, pp. 100-111, Jan. 1994.

[8] J. G. Proakis, Digital Communications. New York: McGraw-Hill, 1995.

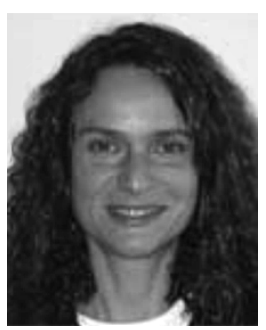

Milica Stojanovic (S'90-M'93) received the B.S. degree from the University of Belgrade, Belgrade, Yugoslavia, in 1988, and the M.S. and Ph.D. degrees in electrical engineering from Northeastern University, Boston, MA, in 1991 and 1993, respectively.

She is currently a Principal Scientist at the Massachusetts Institute of Technology, Cambridge, and also a Guest Investigator at the Woods Hole Oceanographic Institution. Her research interests include digital communications theory and statistical signal processing, and their applications to mobile radio and underwater acoustic communication systems.

Dr. Stojanovic is an Associate Editor for Communications for the IEEE TRANSACTIONS ON Vehicular TEChNOLOGY. 